\title{
Association and Path Analysis of Yield and Yield Attributing Traits in Sesame (Sesamum indicum L.)
}

\author{
Bhagwat Singh* and Rjani Bisen
}

Project Co-ordinating Unit (Sesame and Niger), JNKVV, Jabalpur (M.P.), India

*Corresponding author

A B S T R A C T

\section{Keywords \\ Wheat, Correlation coefficient, Path analysis, Direct effect \\ Article Info \\ Accepted: \\ 28 June 2018 \\ Available Online: \\ 10 July 2018}

Correlation and path coefficient analysis were carried out for yield and yield attributing traits in 60 genotypes of sesame during 2018. The genotypic correlation coefficients were found to be of relatively higher magnitude than phenotypic correlation coefficient, indicating strong inherent correlation between traits. The seed yield/plant exhibited positive and significant correlation with days to flower initiation, days to $50 \%$ flowering, number of primary branches per plant, number of secondary branches per plant and number of capsules per plant both at genotypic and phenotypic levels. Path analysis revealed that, days to flower initiation and number of capsules per plant exhibited highest direct effect on seed yield per plant. This suggests that selection for this component characters can help in improvement of seed yield in sesame.

\section{Introduction}

Sesame (Sesamum indicum L.) is an annual plant that belongs to the Pedaliaceae family. It is an erect herbaceous annual plant with either single stemmed or branched growth habits and two growth characteristics of indeterminate and determinate, reaching up to $2 \mathrm{~m}$ height and with a large tap root of $90 \mathrm{~cm}$ (Pham et at, 2010). Most of the sesame seeds which are rich in fat protein, carbohydrates, fibre and some minerals are used for oil extraction and the rest are used for edible purposes (El Khier et at, 2008). Among the different varieties of sesame Sesamum indicum is the most usually cultivated variety all over the world. Sesame which is grown for its seeds contains about $50-60 \%$ oil content is also rich in fat, protein, carbohydrates, fibre and some minerals (Caliskan et ah, 2004).

India holds a premier position in the global oilseeds scenario accounting for 29 per cent of the total area and 26 per cent of production.In India, sesame is cultivated in 17.138 lakh hectare with a production of 7.84 lakh tonnes and productivity of $457 \mathrm{~kg} / \mathrm{ha}$. Madhya Pradesh contributes $19.71 \%$ and $23.68 \%$ share of country's area (3.80 lakh ha) and production (1.94 lakh tonnes), respectively with productivity of $511 \mathrm{Kg} / \mathrm{ha}$ (DACNET 2016-17). 
Correlation analysis is a statistical measure used to measure the degree and direction of relationship between two or more variables. Most of the characters of interest to breeders are complex and are the result of the interaction of a number of components. Understanding the relationship between yield and its components is of paramount importance for making the best use of these relationships in selection. Character association derived by correlation coefficient,forms the basis for selecting the desirable plant, aiding in evaluation of relative influence of various component characters on seed yield.

The path coefficient analysis is simply a standardized partial regression coefficient which splits the correlation coefficient into the measure of direct and indirect effects. The concept of path coefficient was developed by Wright (1921). Path coefficient analysis was applied for assessment by Dewey and $\mathrm{Lu}$ (1959) in crested wheat grass.

The path analysis unravels whether the association of independent characters with dependent variable is due to their direct effect on it or is a consequence of their indirect effect via some other traits. If the correlation between dependent and independent variables is due to their direct effects of the character, it reflects a true relationship between them and selection can be practiced for such character in order to improve the dependent variable. But, if the association is mainly through indirect effect of the character via another component character, then the breeder has to select for the latter through which the indirect effect is exerted.

\section{Materials and Methods}

The present investigation will be conducted during Summer 2018 under Project Coordinating Unit (Sesame and Niger)
Research Farm, JNKVV, Jabalpur in Randomized Complete Block Design with three replications. The experimental material consisted of 60 sesame genotypes. Observations were recorded on five randomly selected competitive plants for yield and yield attributing traits.

\section{Statistical analysis}

\section{Correlation coefficient}

The Correlation coefficients were calculated to determine the degree of association of the characters with yield and also among the different yield attributing traits. Phenotypic coefficient of correlation between all pairs of characters was determined by using variance and covariance components as suggested by Al-Jibouri at al. (1958). The analysis was done using the Window-stat computer programme.

\section{Path coefficient analysis}

Path coefficient analysis was done using the correlation coefficients to ascertain the direct and indirect effects of the yield components on yield as suggested by Wright (1921) and illustrated by Dewey and Lu (1959). The analysis was done using the Window-stat computer programme.

\section{Results and Discussion}

Correlation coefficient among yield and yield attributing traits

Correlation coefficient helps the breeder in determining relative importance of yield components for indirect selection for yield. Understanding the relationships among yield and yield components is of Paramount importance for making the best use of these relationships In selection. Results indicates that higher values of genotypic correlation coefficients than corresponding phenotypic 
correlation coefficients indicating a low Influence of environmental factors.

Days to flower initiation showed highly significant positive association with days to $50 \%$ flowering (0.9866) and seed yield per plant (0.1947) and significant positively associated with number of capsule per plant (0.1786), days to $50 \%$ flowering showed highly significant positive association with seed yield per plant (0.2109) and number of capsule per plant (0.1981), plant height showed highly significant negative association with 1000 seed weight $(-0.2325)$, number of primary branches per plant showed highly significant positive association with seed yield per plant (0.2085) and number of capsule per plant (0.2112), number of secondary branches per plant showed significant positive association with seed oil content (0.1801), number of capsule per plant (0.1666), number of capsule per plant showed highly significant positive association with seed yield per plant (0.9102), number of seed per capsule showed highly significant positive association with oil content (0.1969) and significant negatively associated with seed yield per plant $(-0.1586)$ and 1000 seed weight significant positively associated with oil content (0.1858). Indicating that these characters can be considered for selection for higher yield, as these were mutually and directly associated with grain yield/plant.

Path coefficient analysis of different traits contributing towards seed yield per plant showed that days to flower initiation (2.618) had highest positive direct effect followed by number of capsule per plant (0.807), plant height (0.308), oil content (0.279), capsule length (0.273), harvest index (0.224), and number of secondary branches per plant (0.131), however, days to $50 \%$ flowering (2.326), number of seed per capsule (-0.359) and number of primary branches per plant (0.235 ) exhibited negative direct effect. All other direct effect was of mostly negative and low in magnitude. Days to flower initiation had maximum positive indirect effect on seed yield per plant through days to $50 \%$ flowering (2.604) followed by number of capsule per plant (0.832) and number of primary branches per plant (0.542), While, it had negative indirect effect on seed yield per plant via plant height (-1.029), harvest index (-0.514), 1000 seed weight $(-0.346)$, capsule length $(-0.323)$ and number of secondary branches per plant (0.289 ). Days to $50 \%$ flowering had maximum positive indirect effect on seed yield per plant through plant height (1.013) followed by harvest index (0.439), number of secondary branches per plant (0.267), number of seed per capsule (0.266), 1000 seed weight (0.259) and capsule length (0.237), While, it had negative indirect effect on seed yield per plant via days to flower initiation (-2.314), number of capsule per plant $(-0.843)$ and number of primary branches per plant $(-0.420)$. Plant height had maximum positive indirect effect on seed yield per plant through days to maturity (0.151), While, it had negative indirect effect on seed yield per plant via days to $50 \%$ flowering (-0.134) and 1000 seed weight (-0.131). Number of capsule/ plant had maximum positive indirect effect on seed yield per plant through days to $50 \%$ flowering (0.293), days to flower initiation (0.257), 1000 seed weight (0.182), number of primary branches per plant (0.178), number of secondary branches per plant (0.133) and days to maturity (0.112), While, it had negative indirect effect on seed yield per plant via number of seed per capsule (-0.215).

Considering the correlation coefficient and path coefficient analysis for grain yield and yield components, an ideal plant type in sesame would be one with days to flower initiation and number of capsules per plant. Therefore, more emphasis should be given to these components while making selection for development of high yielding wheat varieties in future. 
Table.1 List of genotypes used in study

\begin{tabular}{|c|c|c|c|}
\hline S.No. & Genotypes & S.No. & Genotypes \\
\hline 1 & RT-127 & 31 & TMV-7 \\
\hline 2 & RT-346 & 32 & THILAK \\
\hline 3 & RT-103 & 33 & PKV-NT-11 \\
\hline 4 & RT-54 & 34 & YLM-17 \\
\hline 5 & RT-46 & 35 & YLM-66 \\
\hline 6 & GT-1 & 36 & THILARANI \\
\hline 7 & GT-2 & 37 & $\mathrm{CO}-1$ \\
\hline 8 & TKG-306 & 38 & RT-125 \\
\hline 9 & JTS-8 & 39 & RT-351 \\
\hline 10 & TKG-55 & 40 & GT-10 \\
\hline 11 & TKG-22 & 41 & BRIJESHWARI \\
\hline 12 & JLT-408 & 42 & RAJESHWARI \\
\hline 13 & MT-75 & 43 & DSS-9 \\
\hline 14 & VRI-1 & 44 & VINAYAK \\
\hline 15 & VRI-2 & 45 & TARUN \\
\hline 16 & DS-5 & 46 & KALIKA \\
\hline 17 & PT-1 & 47 & KRISHNA \\
\hline 18 & $\mathrm{~N}-32$ & 48 & HIMA \\
\hline 19 & RAMA & 49 & THILATHARA \\
\hline 20 & GT-4 & 50 & $\mathrm{~N}-8$ \\
\hline 21 & $\mathrm{~T}-4$ & 51 & DS-1 \\
\hline 22 & AKT-101 & 52 & YLM-11 \\
\hline 23 & NIRMALA & 53 & SEKHAR \\
\hline 24 & USHA & 54 & JLT-7 \\
\hline 25 & CHANDANA & 55 & PRACHI \\
\hline 26 & SMARK & 56 & TMV-4 \\
\hline 27 & SAVITRI & 57 & KANAK \\
\hline 28 & PKDS-8 & 58 & SSD-5 \\
\hline 29 & PKDS-11 & 59 & JT-12(PKDS-12) \\
\hline 30 & SWETHA TILL-1 & 60 & TKG-21 \\
\hline
\end{tabular}


Table.2 Phenotypic and Genotypic correlation analysis for yield and yield related traits in sesame

\begin{tabular}{|c|c|c|c|c|c|c|c|c|c|c|c|c|c|c|}
\hline Character & & $\begin{array}{c}\text { Days to } \\
\text { flower } \\
\text { initiation }\end{array}$ & $\begin{array}{c}\text { Days to } \\
50 \% \\
\text { flowering }\end{array}$ & $\begin{array}{l}\text { Days to } \\
\text { maturity }\end{array}$ & $\begin{array}{c}\text { Capsule } \\
\text { length } \\
(\mathrm{cm})\end{array}$ & $\begin{array}{c}\text { Plant } \\
\text { height } \\
\text { (cm) }\end{array}$ & $\begin{array}{c}\text { Number of } \\
\text { primary } \\
\text { branches } \\
\text { per plant }\end{array}$ & $\begin{array}{c}\text { Number of } \\
\text { secondary } \\
\text { branches per } \\
\text { plant }\end{array}$ & $\begin{array}{c}\text { Number of } \\
\text { capsule/ } \\
\text { plant }\end{array}$ & $\begin{array}{c}\text { number of } \\
\text { seed per } \\
\text { capsule }\end{array}$ & $\begin{array}{l}1000 \text { seed } \\
\text { weight }(g)\end{array}$ & $\begin{array}{c}\text { Oil } \\
\text { content } \\
(\%)\end{array}$ & $\begin{array}{c}\text { Harvest } \\
\text { index }\end{array}$ & $\begin{array}{c}\text { Seed yield } \\
\text { per plant }\end{array}$ \\
\hline \multirow{2}{*}{$\begin{array}{c}\text { Days to } \\
\text { flower } \\
\text { initiation }\end{array}$} & G & & 0.9948 & -0.039 & -0.1235 & -0.3931 & 0.207 & -0.1104 & 0.3178 & -0.0705 & -0.1322 & -0.0209 & -0.1964 & 0.3208 \\
\hline & $\mathrm{P}$ & & $0.9866 * * *$ & 0.0913 & 0.0249 & -0.0877 & 0.0341 & -0.0751 & $0.1786 *$ & -0.102 & -0.1112 & -0.0827 & 0.0052 & $0.1947 * *$ \\
\hline \multirow{2}{*}{$\begin{array}{c}\text { Days to } 50 \% \\
\text { flowering }\end{array}$} & $\mathrm{G}$ & & & 0.0082 & -0.1018 & -0.4353 & 0.1804 & -0.1147 & 0.3626 & -0.1143 & -0.1111 & 0.0163 & -0.1889 & 0.3556 \\
\hline & $\mathrm{P}$ & & & 0.1109 & 0.0339 & -0.0883 & 0.0238 & -0.0724 & $0.1981 * *$ & -0.1152 & -0.1139 & -0.0724 & 0.0138 & $0.2109 * *$ \\
\hline \multirow{2}{*}{$\begin{array}{l}\text { Days to } \\
\text { maturity }\end{array}$} & $\mathrm{G}$ & & & & -0.1241 & 0.4904 & -0.2485 & 0.0028 & 0.1387 & -0.0181 & -0.0904 & -0.1734 & 0.1505 & 0.1211 \\
\hline & $\mathrm{P}$ & & & & -0.0536 & 0.0452 & -0.0387 & -0.0106 & -0.0422 & -0.0269 & -0.0989 & -0.0816 & -0.0279 & -0.0274 \\
\hline \multirow{2}{*}{$\begin{array}{l}\text { Capsule } \\
\text { length }(\mathrm{cm}\end{array}$} & $\mathrm{G}$ & & & & & 0.1397 & 0.0539 & 0.1046 & 0.0566 & 0.3652 & -0.0795 & -0.1353 & -0.0041 & 0.1118 \\
\hline & $\mathrm{P}$ & & & & & -0.0295 & 0.0317 & 0.1239 & 0.0324 & 0.0923 & -0.0441 & -0.0928 & -0.0266 & 0.0611 \\
\hline \multirow{2}{*}{$\begin{array}{c}\text { Plant height } \\
(\mathrm{cm}\end{array}$} & G & & & & & & 0.1859 & -0.1322 & -0.0874 & -0.0809 & -0.4249 & -0.2397 & -0.2686 & 0.0852 \\
\hline & $\mathrm{P}$ & & & & & & 0.003 & -0.0364 & -0.0505 & -0.0591 & $-0.2325 * *$ & -0.1181 & 0.018 & 0.0024 \\
\hline \multirow{2}{*}{$\begin{array}{c}\text { Number of } \\
\text { primary } \\
\text { branches per } \\
\text { plant }\end{array}$} & G & & & & & & & 0.0837 & 0.2211 & -0.0978 & -0.4493 & -0.0091 & 0.0559 & 0.2073 \\
\hline & $\mathrm{P}$ & & & & & & & 0.1406 & $0.2112 * *$ & -0.0658 & -0.1403 & 0.0471 & 0.0316 & $0.2085 * *$ \\
\hline \multirow{2}{*}{$\begin{array}{c}\text { Number of } \\
\text { secondary } \\
\text { branches per } \\
\text { plant }\end{array}$} & $\mathrm{G}$ & & & & & & & & 0.1642 & 0.3406 & 0.0177 & 0.244 & -0.053 & 0.1427 \\
\hline & $\mathrm{P}$ & & & & & & & & 0.1316 & $0.1666 *$ & -0.0039 & $0.1801 *$ & -0.048 & 0.1203 \\
\hline \multirow{2}{*}{$\begin{array}{c}\text { Number of } \\
\text { capsule/ plant }\end{array}$} & $\mathrm{G}$ & & & & & & & & & -0.2661 & 0.226 & 0.0763 & 0.2358 & 0.916 \\
\hline & $\mathrm{P}$ & & & & & & & & & -0.1224 & 0.1302 & 0.0709 & 0.1168 & $0.9102 * * *$ \\
\hline \multirow{2}{*}{$\begin{array}{c}\text { number of } \\
\text { seed per } \\
\text { capsule }\end{array}$} & $\mathrm{G}$ & & & & & & & & & & 0.0615 & 0.3821 & -0.1102 & -0.2684 \\
\hline & $\mathrm{P}$ & & & & & & & & & & 0.0032 & $0.1969 * *$ & -0.0471 & $-0.1586 *$ \\
\hline \multirow{2}{*}{$\begin{array}{l}1000 \text { seed } \\
\text { weight }(\mathrm{g}\end{array}$} & G & & & & & & & & & & & 0.3492 & -0.1301 & 0.0907 \\
\hline & $\mathrm{P}$ & & & & & & & & & & & $0.1858 *$ & -0.0211 & 0.0566 \\
\hline \multirow{2}{*}{$\begin{array}{l}\text { Oil content } \\
(\%)\end{array}$} & $\mathrm{G}$ & & & & & & & & & & & & -0.1657 & 0.0005 \\
\hline & $\mathrm{P}$ & & & & & & & & & & & & -0.0837 & -0.0119 \\
\hline \multirow[t]{2}{*}{ Harvest index } & $\mathrm{G}$ & & & & & & & & & & & & & 0.2244 \\
\hline & $\mathrm{P}$ & & & & & & & & & & & & & 0.1158 \\
\hline \multirow{2}{*}{$\begin{array}{l}\text { Seed yield } \\
\text { per plant }\end{array}$} & $\mathrm{G}$ & & & & & & & & & & & & & \\
\hline & $\mathrm{P}$ & & & & & & & & & & & & & \\
\hline
\end{tabular}


Table.3 Genotypic path coefficient analysis showing direct and indirect effects for yield and yield related traits in sesame

\begin{tabular}{|c|c|c|c|c|c|c|c|c|c|c|c|c|}
\hline Character & $\begin{array}{c}\text { Days to } \\
\text { flower } \\
\text { initiation }\end{array}$ & $\begin{array}{c}\text { Days to } \\
50 \% \\
\text { Flowering }\end{array}$ & $\begin{array}{l}\text { Days to } \\
\text { Maturity }\end{array}$ & $\begin{array}{c}\text { Capsule } \\
\text { Length } \\
(\mathrm{cm})\end{array}$ & $\begin{array}{c}\text { Plant } \\
\text { Height } \\
\text { (cm) }\end{array}$ & $\begin{array}{c}\text { Number of } \\
\text { primary } \\
\text { branches } \\
\text { per plant }\end{array}$ & $\begin{array}{c}\text { Number } \\
\text { of } \\
\text { secondary } \\
\text { branches } \\
\text { per plant }\end{array}$ & $\begin{array}{c}\text { Number } \\
\text { of } \\
\text { capsule/ } \\
\text { plant }\end{array}$ & $\begin{array}{c}\text { number } \\
\text { of seed } \\
\text { per } \\
\text { capsule }\end{array}$ & $\begin{array}{c}1000 \\
\text { seed } \\
\text { weight } \\
(\mathrm{g})\end{array}$ & $\begin{array}{c}\text { Oil } \\
\text { content } \\
(\%)\end{array}$ & $\begin{array}{c}\text { harvest } \\
\text { index }\end{array}$ \\
\hline Days to flower initiation & 2.618 & 2.604 & -0.102 & -0.323 & -1.029 & 0.542 & -0.289 & 0.832 & -0.185 & -0.346 & -0.055 & -0.514 \\
\hline Days to $50 \%$ flowering & -2.314 & -2.326 & -0.019 & 0.237 & 1.013 & -0.420 & 0.267 & -0.843 & 0.266 & 0.259 & -0.038 & 0.439 \\
\hline Days to maturity & 0.002 & 0.000 & -0.038 & 0.005 & -0.019 & 0.009 & 0.000 & -0.005 & 0.001 & 0.003 & 0.007 & -0.006 \\
\hline Capsule length $(\mathrm{cm})$ & -0.034 & -0.028 & -0.034 & 0.273 & 0.038 & 0.015 & 0.029 & 0.015 & 0.100 & -0.022 & -0.037 & -0.001 \\
\hline Plant height $(\mathrm{cm})$ & -0.121 & -0.134 & 0.151 & 0.043 & 0.308 & 0.057 & -0.041 & -0.027 & -0.025 & -0.131 & -0.074 & -0.083 \\
\hline Number of primary branches per plant & -0.049 & -0.042 & 0.058 & -0.013 & -0.044 & -0.235 & -0.020 & -0.052 & 0.023 & 0.106 & 0.002 & -0.013 \\
\hline Number of secondary branches per plant & -0.014 & -0.015 & 0.000 & 0.014 & -0.017 & 0.011 & 0.131 & 0.021 & 0.045 & 0.002 & 0.032 & -0.007 \\
\hline Number of capsule/ plant & 0.257 & 0.293 & 0.112 & 0.046 & -0.071 & 0.178 & 0.133 & 0.807 & -0.215 & 0.182 & 0.062 & 0.190 \\
\hline number of seed per capsule & 0.025 & 0.041 & 0.007 & -0.131 & 0.029 & 0.035 & -0.122 & 0.096 & -0.359 & -0.022 & -0.137 & 0.040 \\
\hline 1000 seed weight $(\mathrm{g})$ & 0.001 & 0.001 & 0.001 & 0.001 & 0.004 & 0.004 & 0.000 & -0.002 & -0.001 & -0.009 & -0.003 & 0.001 \\
\hline Oil content $(\%)$ & -0.006 & 0.005 & -0.048 & -0.038 & -0.067 & -0.003 & 0.068 & 0.021 & 0.107 & 0.098 & 0.279 & -0.046 \\
\hline Harvest index & -0.044 & -0.042 & 0.034 & -0.001 & -0.060 & 0.013 & -0.012 & 0.053 & -0.025 & -0.029 & -0.037 & 0.224 \\
\hline Seed yield per plant & 0.321 & 0.356 & 0.121 & 0.112 & 0.085 & 0.207 & 0.143 & 0.916 & -0.268 & 0.091 & 0.001 & 0.224 \\
\hline
\end{tabular}


Fig.1 Genotypic path for seed yield per plant

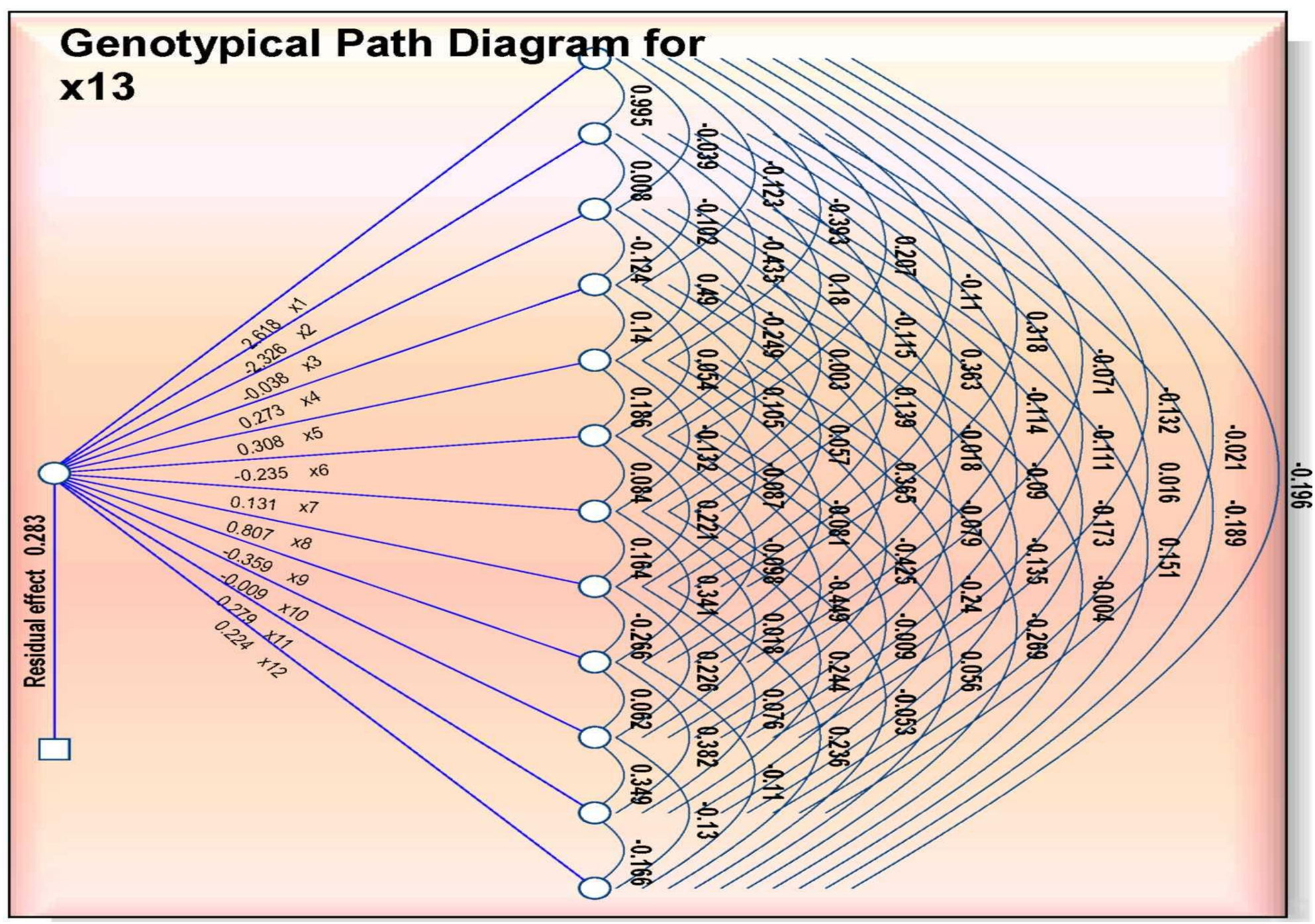




\section{References}

Al-Jibouri H. A., Miller P. A. and Robinson H. F. (1958). Genotypic and environmental co-variances in an upland cotton cross of inter specific origin. Agron. J., 50:633-637.

Caliskan S, Arslan M, Arioglu and Isler N. 2004. Effect of planting method and plant population on growth and yield of sesame (Sesamum indicum L.) in a Mediterranean type of environments. Asian J. Plant Sci., 3:610-613.

DACNET 2016-17. 4th Advanced Estimation. GOI.

Dewey DR and Lu KH. 1959. A correlation and path coefficient analysis of components of crested wheat grass seed production. Agronomy Journal 51 (9): 515-518.

EL Khier MKS, Ishag KEA and Yagoub AEA. 2008. Chemical composition and oil characteristics of sesame seed cultivars grown in Sudan. Res. J. Agric. Biol. Sci., 4:761-766.

Pham TD, Nguyen T, Carlsson AS and Bui TM. 2010. Morphological evaluation of sesame (Sesamum indicum L.) varieties from different origins. Aust. J. Crop Sci., 4:498-504.

Wright S. 1921. Correlation and causation. Journal of Agriculture Research 20 : 557-585.

\section{How to cite this article:}

Bhagwat Singh and Rjani Bisen. 2018. Association and Path Analysis of Yield and Yield Attributing Traits in Sesame (Sesamum indicum L.). Int.J.Curr.Microbiol.App.Sci. 7(07): 40414048. doi: https://doi.org/10.20546/ijcmas.2018.707.470 